\title{
Extraction mechanism of lithium from the alkali solution with diketonate-based ionic liquid extractants
}

\author{
Junfeng Wang ${ }^{a} b^{*}$, Shicheng Yang ${ }^{a, b}$, Xiaofei Zhang ${ }^{c}$, Yilin Wang ${ }^{c}$, Daoguang Wang ${ }^{a, b}$, Weichao Li ${ }^{a, b}$,

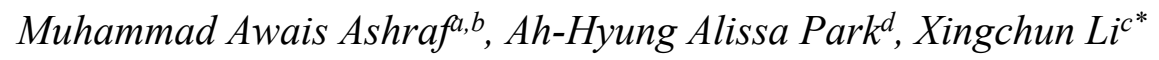

${ }^{a}$ Beijing Key Laboratory of Ionic Liquids Clean Process, State Key Laboratory of Multiphase Complex Systems, Institute of Process Engineering, Chinese Academy of Sciences, Beijing 100190, PR China

${ }^{\mathrm{b}}$ Innovation Academy for Green Manufacture, Chinese Academy of Sciences, Beijing 100190, PR China

${ }^{\mathrm{c}}$ State Key Laboratory of Petroleum Pollution Control, CNPC Research Institute of Safety and Environmental Technology, Beijing 102206, PR China

${ }^{\mathrm{d}}$ Departments of Earth \& Environmental Engineering and Chemical Engineering, Lenfest Center for Sustainable Energy, Columbia University, 500 W. 120th Street, New York, NY 10027

*Corresponding Authors: Tel.: +86 10 82627080. Fax: +86 10 82627080. E-mail: junfwang@ipe.ac.cn, li-xingchun@enpc.com.cn. 
Table S1. Purities of ILs and their separation factor $\left(\beta_{\mathrm{Li} / \mathrm{Na}}\right)$ by IL+TRPO with different initial pH of aqueous solution at: $\mathrm{IL}=0.5 \mathrm{~mol} \cdot \mathrm{L}^{-1}, \mathrm{~A} / \mathrm{O}=1: 1$, and $\mathrm{T}=298.15 \mathrm{~K}$.

\begin{tabular}{cccccc}
\hline \multirow{2}{*}{ ILs } & Purity & \multicolumn{4}{c}{ Separation factors $\left(\boldsymbol{\beta}_{\mathrm{Li} / \mathrm{Na}}\right)$} \\
\cline { 3 - 6 } & $\mathbf{( \% )}$ & $\mathbf{p H = 1 . 2 6}$ & $\mathbf{p H = 6 . 5 6}$ & $\mathbf{p H = 1 0 . 1 7}$ & $\mathbf{p H = 1 3 . 1}$ \\
& & & & & $\mathbf{1}$ \\
\hline$[\mathrm{A} 336]$ BTA & 95.12 & 15.07 & 47.54 & 125.09 & 275.95 \\
{$[$ A336]TTA } & 97.65 & 35.37 & 76.45 & 160.53 & 284.04 \\
{$[$ A336]TFBA } & 96.83 & 7.90 & 30.86 & 97.90 & 212.38 \\
{$[$ A336]TTBA } & 97.26 & 3.84 & 16.48 & 60.37 & 190.80 \\
\hline
\end{tabular}

Table S2. Standard-state chemical potentials and enthalpies of formation of species.

\begin{tabular}{cccc}
\hline Species & $-\Delta \mu_{i}^{0} /\left(\mathbf{k J} \cdot \mathbf{m o l} \mathbf{- 1}^{-1}\right)$ & $-\Delta_{f} H_{i}^{0} /\left(\mathbf{k J} \cdot \mathbf{m o l}{ }^{-1}\right)$ & Ref. \\
& & & \\
\hline $\mathrm{Li}^{+}(\mathrm{aq})$ & 293.80 & 278.50 & {$[37,38]$} \\
$\mathrm{CO}_{3}{ }^{2-}(\mathrm{aq})$ & 528.10 & 676.20 & {$[37,38]$} \\
$\mathrm{Li}_{2} \mathrm{CO}_{3}(\mathrm{~s})$ & 1133.00 & 1216.00 & {$[37,38]$} \\
\hline
\end{tabular}

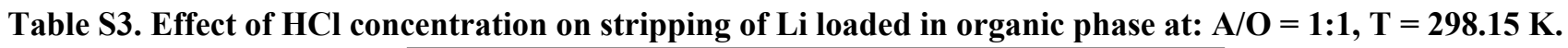

\begin{tabular}{ccc}
\hline $\begin{array}{c}\text { HCl concentration } \\
\left(\mathbf{m o l} \cdot \mathbf{L}^{-\mathbf{1}}\right)\end{array}$ & \multicolumn{2}{c}{ Stripping percentage (\%) } \\
\cline { 2 - 3 } & $\mathbf{L i}$ & $\mathbf{N a}$ \\
\hline 0.00 & 24.62 & 43.17 \\
0.10 & 49.03 & 61.46 \\
0.20 & 73.64 & 62.20 \\
0.30 & 88.21 & 62.44 \\
0.40 & 93.95 & 63.41 \\
0.50 & 95.59 & 66.10 \\
\hline
\end{tabular}


Table S4. Thermodynamic parameters of extracting Li by [A336]TTA.

\begin{tabular}{ccc}
\hline Temperature $(\mathbf{K})$ & $\Delta \boldsymbol{H}(\mathbf{K J} / \mathbf{m o l})$ & $\Delta \boldsymbol{G}(\mathbf{K J} / \mathbf{m o l})$ \\
\hline 298.15 & & -7.97 \\
303.15 & & -6.83 \\
313.15 & -10.63 & -6.55 \\
323.15 & & -6.52 \\
333.15 & -6.38 \\
343.15 & -6.19 \\
\hline
\end{tabular}


Table S5. Compositions of the mother solution used in section 3.6.

\begin{tabular}{cc}
\hline Components & Content $/\left(\mathbf{g} \cdot \mathbf{L}^{-1}\right)$ \\
\hline $\mathrm{Li}$ & 1.41 \\
$\mathrm{Ca}$ & $3.29 \times 10^{-3}$ \\
$\mathrm{Mg}$ & $1.94 \times 10^{-7}$ \\
$\mathrm{Na}$ & 63.23 \\
$\mathrm{Cl}$ & 73.79 \\
$\mathrm{SO}_{4}$ & 7.83 \\
$\mathrm{CO}_{3}$ & 16.76 \\
$\mathrm{~B}_{2} \mathrm{O}_{3}$ & 0.00 \\
\hline
\end{tabular}




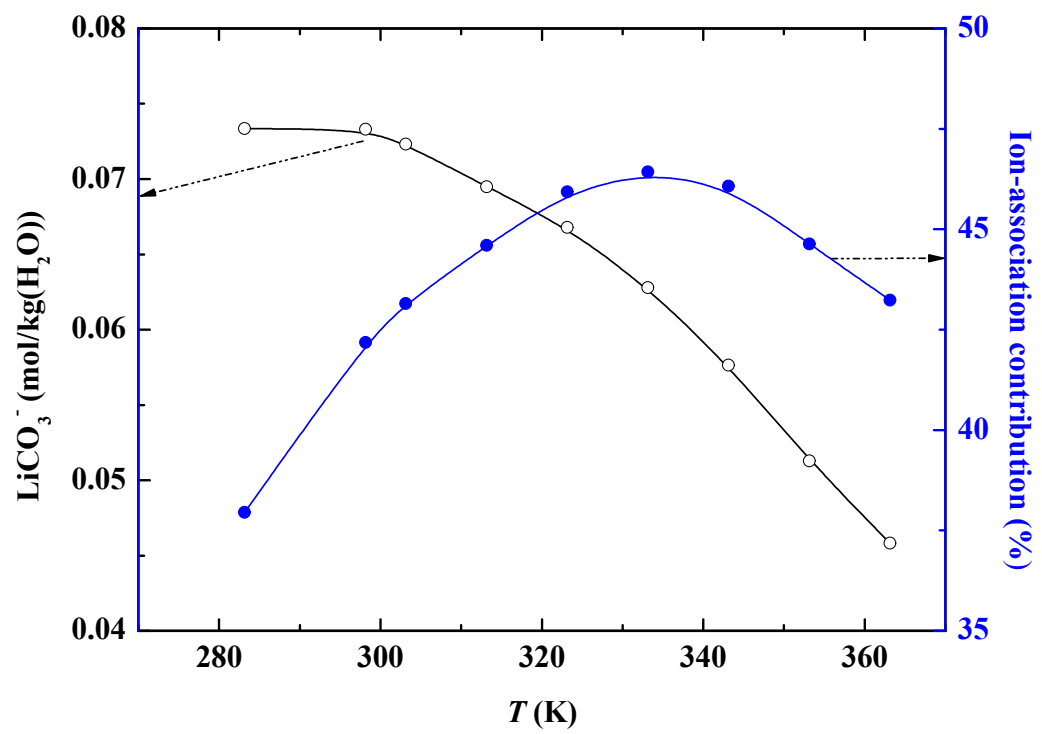

Figure S1. Relationships of $\mathrm{LiCO}_{3}{ }^{-}$concentration and ion-association contribution with temperatures. 